\title{
Design, manufacture and testing of the glow discharge electrodes for Wendelstein 7-X
}

\author{
A. Spring ${ }^{1}$, A. Cardella ${ }^{2}$, B. Brucker ${ }^{3}$, B. Mendelevitch ${ }^{3}$, H. Niedermeyer ${ }^{1}$, O. \\ Sellmeier ${ }^{3}$, S. Schweizer ${ }^{3}$, B. Streibl ${ }^{3}$ \\ ${ }^{1}$ Max-Planck-Institut für Plasmaphysik, Euratom Association, \\ Teilinstitut Greifswald, Wendelsteinstr. 1, D-17491 Greifswald, Germany \\ ${ }^{2}$ European Commission, clo W-7X, Boltzmannstr. 2, D-85742 Garching, Germany \\ ${ }^{3}$ Max-Planck-Institut für Plasmaphysik, Euratom Association, \\ Boltzmannstr. 2, D-85742 Garching, Germany \\ Corresponding author: anett.spring@ipp.mpg.de
}

The electrodes for the Wendelstein 7-X glow discharge system have been designed, tested and manufactured. The compact design relies on a cooled housing, integrated into the first wall cooling system, and a calotte-shaped graphite anode. The new mounting concept avoids the need of active cooling of the anode due to an improved thermal conduction. Comprehensive tests of a prototype electrode had been carried out in laboratory and in the ASDEX Upgrade Tokamak during two operation campaigns. The electrode showed excellent and reliable longtime discharge behavior and fulfilled all the requirements regarding temperature limits and maintainability resulting from the steady-state operation of W7-X.

Keywords: Wendelstein 7-X; glow discharge electrodes; wall conditioning.

\section{Background}

The fusion device Wendelstein 7-X will need conditioning by glow discharges during commissioning, after shutdown phases and during experiment breaks. The W7-X glow discharge system has been developed as a low-maintenance and space-saving system for operation in a steady-state fusion experiment. Ten electrodes will be integrated into the first wall [FIG.1], approximately equidistant on the vessel circumference. The innovative electrodes' geometry consists of a calotte-shaped graphite anode mounted, electrically insulated, in a stainless steel housing. In this regard the W7-X glow electrode design differs basically from those at other fusion devices, e.g. ASDEX Upgrade [1], JET [2], LHD [3] or ITER [4].

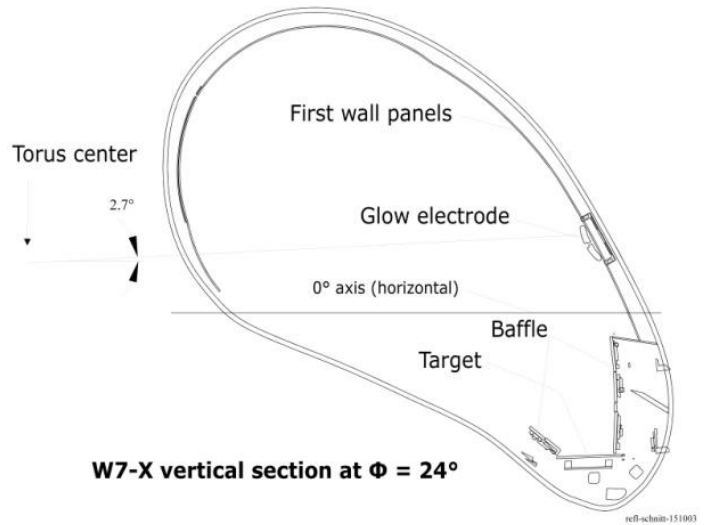

Figure 1: Position of the glow electrodes integrated into the first wall of W7-X. 
Comprehensive experiments in the W7-X DEMO plasma vessel have been carried out to optimize the electrode's shape, dimension and position relatively to the housing and to study the long-time behavior. As a result a first prototype has been tested successfully both regarding the discharge performance and the electrodes' thermo-mechanic reliability [5].

\section{Requirements at the W7-X steady state device}

Based upon the prototype tests the detailed design and construction of the electrodes has been further developed to meet the requirements coming from machine operation restrictions and diagnostics constraints:

a. The heat load onto the vessel wall during the glow discharge has to be limited. Depending on the discharge conditions, namely discharge current and gas pressure, the anode will heat up. The cooling system for the cryostat vessel wall is designed for a maximum external heat load of $2 \mathrm{~kW} / \mathrm{m}^{2}$. Because it is planned to execute glow discharge cleaning in presence of a cooled cryostat, this limit must not be exceeded.

\section{b. The anode's temperature during stellarator plasma operation has to be minimized.}

The thermal load onto the anode caused by the steady state stellarator plasma onto the first wall at the designated position is estimated to be up to $50 \mathrm{~kW} / \mathrm{m}^{2}$. The resulting heating and subsequent thermal radiation of the glow anode may disturb infrared-diagnostics. This has to be avoided to a large extent.

c. The limited machine access demands efficient maintenance of the electrodes. The electrodes' design has to be focused on reliability and maintenance as simple as possible. The parts of the electrodes which need cleaning or repair have to be easily exchangeable.

\section{Design enhancement}

The first two requirements imply the need of sufficient cooling, the third the optimization of the prototype design and mounting concept. The approach and successful implementation will be presented here.

\subsection{Cooling}

The cooling requirements are fulfilled with two main design solutions: active cooling of the housing and enhanced thermal heat conduction from the anode to the housing by redesigning the mounting concept - in contrast to the first prototype which relied mainly on radiation cooling.

Measurements at the DEMO cryostat during a long-term glow discharge with an un-cooled housing showed a maximum temperature of about $400^{\circ} \mathrm{C}$ at the bottom. The new housing [FIG.2] construction allows the integration into the central cooling of the in-vessel components. It is made out of a cylindrical stainless steel form with a carved-in cooling channel. The bottom side is closed with a steel plate attached by electron-beam welding. The channel dimensions have been calculated to ensure sufficient cooling capacity and to fulfill the requirements regarding water flow and pressure loss. The connection tubes will be welded to the in-vessel components cooling system. 

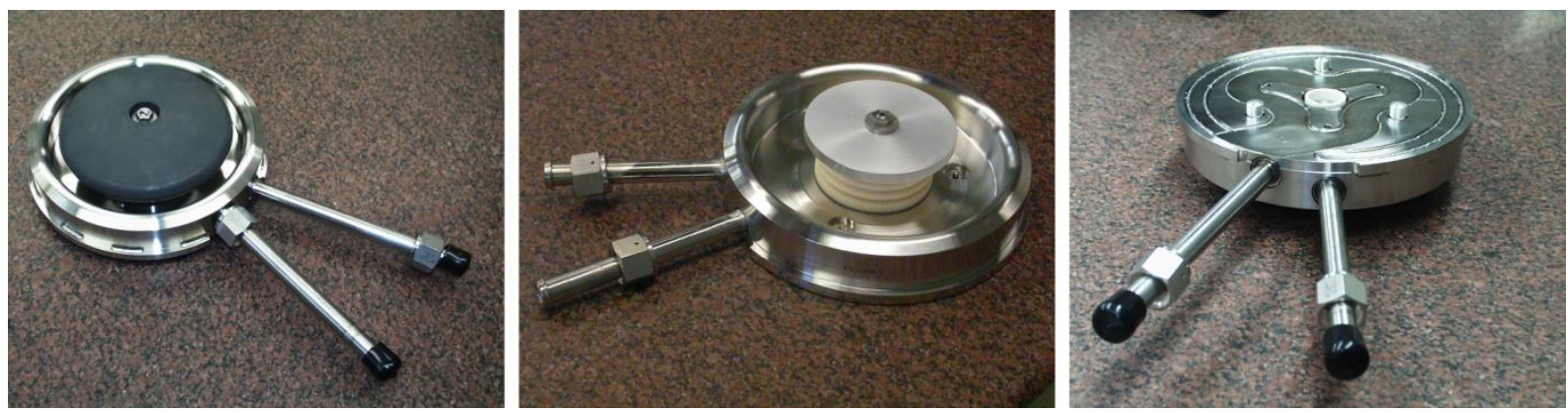

Figure 2: The W7-X glow electrode: top, inside and bottom view.

In addition, a new mounting concept [FIG.3] releases the design from the need of an active cooling of the anode. The anode of the first prototype [5] had been mounted using three insulator sockets with only limited thermal contact to the anode. In contrast to this, the new concept is based upon an enhanced thermal contact of the anode's fixation, while guaranteeing the high-voltage insulation: The central support consists of a cylindrical alumina block $\left(\mathrm{Al}_{2} \mathrm{O}_{3}\right)$ with a large contact area to both the hot graphite anode and the cooled housing. A flexible graphite foil on both sides improves the heat transfer. Furthermore, the inside of the housing has been blackened to enhance the thermal radiation absorption.

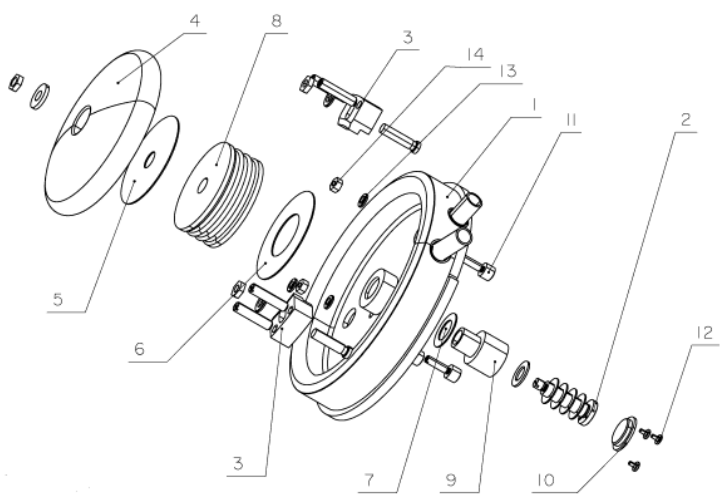

Figure 3: Exploded view of the W7-X glow electrode design with the graphite anode (4), the central alumina insulator $(8)$, graphite foils $(5,6)$ and the water cooled housing (1).

Conservative ANSYS finite element calculations [FIG.4] show that, by these means, the cooling of the anode, which relies on radiation and thermal conduction, ensures the required temperature limitation of the anode. At a maximum heat flux to be expected at the electrodes' position in the plasma vessel of $50 \mathrm{~kW} / \mathrm{m}^{2}$ the temperature at the top of the anode will not exceed $630^{\circ} \mathrm{C}$, sufficient not to disturb diagnostics. Having avoided the active water cooling of the anode, it is not necessary anymore to provide a difficult and risky high-voltage insulation of water pipes. 


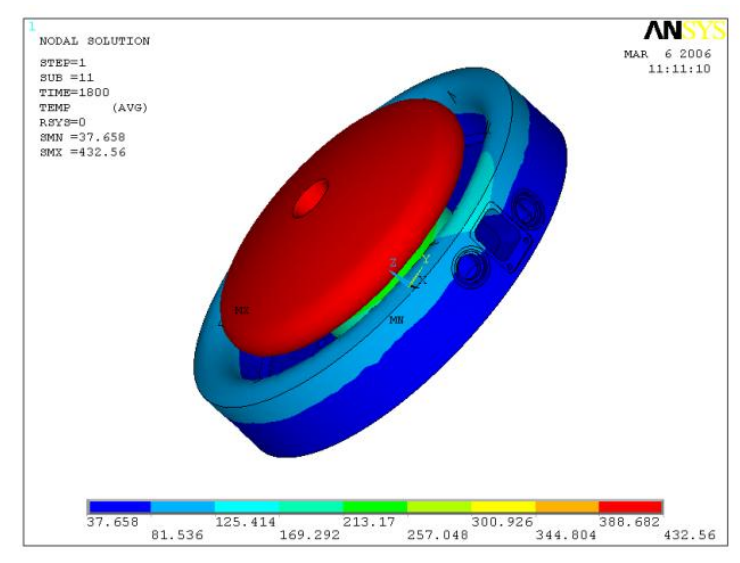

Figure 4: Stationary temperature distribution at the glow electrode calculated for a heat flux of $50 \mathrm{~kW} / \mathrm{m}^{2}$.

\subsection{Maintainability}

To meet the third requirement the electrodes' design has been optimized to allow quick and easy maintenance access to all parts that may need cleaning or repair during their lifetime. This concerns in particular the graphite anode, which is exposed to permanent thermal load, and the insulation, which may get coated with graphite due to sputtering processes during glowing.

Both parts can be dismantled from the front by loosening the central screw and then removing the anode and the alumina including the two graphite foils. All other parts will stay in place. The central screw demands specific attention. Since under operation it exceeds the temperature of the anode, it may be damaged and the connection can become blocked. These problems have been released by using TZM (Titanium-Zirconium-Molybdenum) material. The screw head design has been made in such a way to ensure sufficient torsional moment for tightening and loosening.

As another advantage, the easy mounting concept allows the installation of a dummy anode during the assembly phase of $\mathrm{W} 7-\mathrm{X}$ and to install the degassed anodes right before the machine assembly is completed.

\section{Prototype tests}

A prototype of the final design has been manufactured in IPP in Garching and has undergone a series of extensive tests.

The basic tests included comprehensive leak and pressure tests both at room temperature and at the maximum expected temperature during baking $\left(160^{\circ} \mathrm{C}\right)$, and tests of the electric strength. They were successful and no difficulties occurred.

Pressure loss measurements of the cooling channel in the housing have been carried out at several volume flows. They confirmed the computed values and will serve as a basis for the flow adjustment necessary for the integration into the first wall cooling system.

To verify the enhanced heat flow of the new electrode design, the anode had been exposed to a defined heat load at air while measuring the temperature characteristics at several control points on the insulator and the housing. The results confirm the expected heat transfer as calculated in [FIG.4] and underline the significantly better cooling properties, sufficient to avoid the need of active anode cooling.

Detailed glow tests in a laboratory vessel, including long-term glowing with maximum performance (up to 3A glow current) showed reliable startup and discharge behavior. They verified the results from the first prototype test as well as the heat transfer calculations. Long- 
term discharges had been performed under operating conditions as expected for W7-X glow discharge sessions according to the experiments in [5]: Helium at $0.4 \ldots 1 \mathrm{~Pa}$ and $1.5 \ldots 3 \mathrm{~A}$ (per electrode), resulting in typically $300 \ldots 500 \mathrm{~V}$, depending on pressure. With a watercooled housing and a discharge current of $2 \mathrm{~A}$, the anode's temperature did not exceed $390^{\circ} \mathrm{C}$ at the anode's edge and $270^{\circ} \mathrm{C}$ at the anode's center - thus proving the good heat transfer capability of the central alumina insulator. A series of detailed measurements of the glow discharge parameters under varying conditions, to be published in future, confirmed and enhanced the previous investigations.

As a "real-life" test the prototype electrode has been used successfully in the ASDEX Upgrade Tokamak during two operation campaigns. Used for standard glow discharge conditioning it showed reliable discharge behavior and mechanical long-term stability of all parts and the fixation system in particular. After the last improvements in the fixation of the anode the final demounting of the component was performed easily and failure free.

\section{Status}

The compact electrode's design for W7-X glow discharge has been tested thoroughly and improved. The final prototype passed all tests successfully, thus giving the confidence to have developed a reliable design for the W7-X glow electrodes.

Today the complete set of ten electrodes has been manufactured and verified, the graphite anodes have been degassed and packed separately from the housing. They have been delivered to Greifswald, ready ahead of time to be assembled in W7-X.

\section{Acknowledgements}

The authors would like to thank the colleagues from the IPP Integrated Technical Center, the ASDEX Upgrade team, the student apprentice Thomas Müller, and the W7-X ECRH team for their engagement during the electrode's testing, as well as Minyou Ye for the ANSYS calculations.

\section{References}

[1] W. Poschenrieder, G. Staudenmaier, P. Staib, Conditioning of asdex by glow discharge, Journal of Nuclear Materials, Volumes 93-94, Part 1, October 1980, Pages 322-329, ISSN 0022-3115, DOI: 10.1016/0022-3115(80)90342-6.

[2] G. Saibene, A. Rossi, R. D. Monk, J. Orchard, P. Andrew, R. Barnsley, D. Cushing, P. J. Coad, S. Davies, K. Erents, H. Y. Guo, K. Lawson, J. Lingertat, G. Matthews, G. Sips, M. Stamp, A. Tanga, Review of vacuum vessel conditioning procedures at JET and their impact on plasma operation, Journal of Nuclear Materials, Volumes 220-222, Plasma-Surface Interactions in Controlled Fusion Devices, April 1995, Pages 617-622, ISSN 0022-3115, DOI: 10.1016/0022-3115(94)00551-6.

[3] A. Sagara, M. Iima, S. Inagaki, N. Inoue, H. Suzuki, K. Tsuzuki, S. Masuzaki, et al., Wall Conditioning at the Starting Phase of LHD, Research Report NIFS-586, 1999.

[4] A. B. Antipenkov, P. Ladd, R. Marrs, ITER glow discharge cleaning system, Fusion Engineering and Design, Volumes 56-57, October 2001, Pages 233-238, ISSN 0920-3796, DOI: $10.1016 /$ S0920-3796(01)00264-2.

[5] A. Spring, R. Brakel, H. Niedermeyer, Wall conditioning for Wendelstein 7-X by glow discharge, Fusion Engineering and Design, Volumes 66-68, 22nd Symposium on Fusion Technology, September 2003, Pages 371-375, ISSN 0920-3796, DOI: 10.1016/S09203796(03)00245-X. 